\title{
Tracking quintessence would require two cosmic coincidences
}

\author{
Sidney Bludman* \\ Deutsches Elektronen-Synchrotron DESY, Hamburg, Germany
}

(Received 24 December 2003; published 29 June 2004)

\begin{abstract}
Good tracking requires that the quintessence energy fraction slowly increase while the roll $\lambda \equiv-d \ln V / \varkappa d \phi$ slowly decreases, but is not yet truly slow-rolling. The supernova bound on the present quintessence equation of state requires either (1) a cosmological constant or other fine-tuned "crawling quintessence" or (2) "roll-over quintessence" that tracked until recently, but now became slow rolling, because of a sharp increase in potential curvature. Thus, fine-tuning is required by constant equation of state and inverse power potentials, but can be avoided by the supergravity and Skordis-Albrecht potentials and other good trackers, provided quintessence energy domination and slow roll both began only recently. This makes the time in which we live special in two respects.
\end{abstract}

DOI: 10.1103/PhysRevD.69.122002

PACS number(s): 98.80.Es, 98.80.Cq, 95.35.+d, 98.80.Jk

\section{THE DARK ENERGY DENSITY IS NOW EXACTLY OR NEARLY STATIC}

The most surprising and important recent discovery in cosmology is that the present universe is flat and dominated by unclustered dark energy, whose energy density $\rho_{Q}$ is now small and constant or nearly constant. Indeed, the next few years should show whether this energy density is now truly static (cosmological constant) or slowly evolving with the logarithm of the cosmological scale factor $N \equiv \ln a=-\ln (1$ $+z$ ). If dynamic, we would like to know something about the dynamical equation of state $\gamma_{Q}(N) \equiv-d \ln \rho_{Q} / 3 d N \neq 0$ and the sound speed squared $c_{s}^{2}$. In this section, we review the present bounds on dark energy evolution, before reviewing the constraints on tracking quintessence, a particularly interesting model for the smooth energy in terms of a very low mass canonical scalar field.

\section{A. Kinematics of the accelerating flat FRW universe}

Supernovae Ia and cosmic shear directly explore the space-time geometry by measuring the luminosity distance $d_{L}(z)=(1+z) \eta$ to individual distant supernovae, chosen to be standard candles, and the angular-diameter distance $d_{A}(z)=\eta /(1+z)$ to distant galaxies, from which the comoving distance

$$
\eta \equiv c \int_{z}^{0} d z^{\prime} / H\left(z^{\prime}\right)=c \int_{0}^{t} d t^{\prime} / a\left(t^{\prime}\right)
$$

is inferred. This conformal coordinate distance to the horizon, $\eta$, describes the proper time evolution of the scale factor $a$.

The need for unclustered dark energy derives from general relativity, which is tested to high precision in the classic solar system and binary pulsar observations. General relativity is also confirmed out to cosmological distances and down to ages about one minute by BBN light-element abundances,

\footnotetext{
*Also at University of Pennsylvania, Philadelphia, PA. Email address: bludman@mail.desy.de
}

and is consistent with the observed spectra of primordial density fluctuations, CBR temperature anisotropies, and large-scale structure power. We will therefore ignore alternate gravity theories, such as small-curvature modifications of GR, large extra spatial dimensions or brane cosmology, and assume an homogeneous and isotropic (FriedmannRobertson-Walker) flat universe, the Friedmann expansion rate is

$$
8 \pi G \rho=3 H^{2}, \text { where } H \equiv \dot{a} / a .
$$

Quantum field theory requires that the energy density, and therefore $G$, be positive, so that we can write $\sqrt{8 \pi G} \equiv \varkappa$ $\equiv 1 / M_{P}$, where $M_{P}=2.44 \times 10^{18} \mathrm{GeV}$ is the reduced Planck mass. The derived quantity, the cosmological fluid pressure $P=-d\left(\rho c^{2} a^{3}\right) / d a^{3}$, may be positive or negative.

In terms of geometrical quantities,

$$
\varkappa^{2} P / c^{2}=-\left(2 \dot{H}+3 H^{2}\right),
$$

the enthalpy is

$$
\varkappa^{2}\left(\rho+P / c^{2}\right)=-2 \dot{H}=-d H^{2} / d N=-\left(\varkappa^{2} / 3\right)(d \rho / d N),
$$

and the overall barotropic index is

$$
\gamma \equiv-d \ln \rho / 3 d N=\left(\rho+P / c^{2}\right) / \rho=-\frac{2}{3}(d \ln H / d N) .
$$

Here the logarithm of the cosmological scale factor $N \equiv \ln a$ $=-\ln (1+z)$, so that $d N=H d t$. This equation of state and its quintessence component depends on the Hubble time $H^{-1}$ $=d \eta / d z$, and its derivative $d H^{-1} / d z=d^{2} \eta / d z^{2}$.

In flat FRW cosmology, the space-time curvature (Ricci scalar) is

$$
R \equiv-6\left(\dot{H}+2 H^{2}\right)=\varkappa^{2}(3 P-\rho),
$$

while the acceleration, $\ddot{a} / a=-\varkappa^{2}(\rho+3 P) / 6$, so that

$$
\ddot{a} / \dot{a}^{2}=1-d\left(H^{-1}\right) / d t=-(1+3 w) / 2
$$


ranges from -2 to 1 , when the overall equation of state $w$ $\equiv P / \rho$ ranges from the stiff value 1 down to the ultra-soft value -1 . We now know that, until the recent red-shift $z$ $\sim 0.35$, attractive gravity dominated the cosmological fluid so that large scale structures could form. Only recently, after the expansion rate in Eq. (7) outpaced the growth of the Hubble radius $H^{-1}$, did $P / \rho=<-1 / 3$, and the expansion become accelerated.

\section{B. Present cosmological parameters}

Supernova observations were the first evidence for smooth energy and remain the direct observation of acceleration of the present universe. This supports the possibility of an inflationary early universe, which is otherwise not directly observable.

Because the barotropic index (5) and its quintessence component (13) depend on the first and second derivatives of the comoving distance $\eta$, the quintessence evolution $w_{Q}(z)$ depends on first and second derivatives of the observed luminosity distances [1] or on $H(z), d H(z) / d z$. In practice, quintessence is appreciable only for small red-shift. This means that, before $w_{Q}(z)$ can be extracted from the supernova data, the inherently noisy luminosity distance $d_{L}(z)$ data must be parametrized. For this, and other reasons, along with a large number of high red-shift supernovae, precise knowledge of other cosmological parameters will be needed [2-4], and can still determine only one or two parameters characterizing the potential, such as $w_{Q 0},\left(d w_{Q} / d z\right)_{0}$. A worrisome feature [5] of the present supernova data is that, taken separately, the low $z<0.34$ and high $z>0.34$ observations imply best-fit supernovae Ia absolute magnitudes differing by 0.5 magnitude and are each compatible with an Einstein-Lemaitre $\Omega_{Q 0}=0$. Indeed, the combined data actually favors a supernova-averaged quintessence equation of state

$$
\widetilde{w_{Q}}(N) \equiv \int_{N}^{0} w_{Q}\left(N^{\prime}\right) d N^{\prime} / N<-1 .
$$

While programs to measure luminosity and angular diameter distances are underway, we already know that we live at a time when

$$
\begin{gathered}
\Omega_{Q 0}=0.71 \pm 0.07, \quad \widetilde{w_{Q}}<-0.78 \quad(95 \% \quad \text { C.L. }), \\
h \equiv H_{0} / 100=0.72 \pm 0.05,
\end{gathered}
$$

that the radiation-matter equality took place at red-shift $z_{e q}$ $=3454_{-392}^{+385}[6,7]$, dark energy began dominating over matter $\gtrsim 6.3 \mathrm{Gyr}$ ago, and the cosmological expansion has been accelerating since red-shift $z \sim 0.7[7,8]$. This fit to $\widetilde{w_{Q}}$ derives from SNIa, 2dFGRS and Ly $\alpha$ observations, and assumes $w_{Q}$ constant and $>-1$. Because the observations average over a small range in $z$, in which $w_{Q}(z)$ changes relatively little, $\widetilde{w_{Q}}<-0.78$ is also a rough upper bound to the present value $w_{Q 0}$.

The radiation-matter background density,

$$
\rho_{B}=(11.67 a+0.003378) / a^{4} \mathrm{meV}^{4},
$$

is now $\rho_{B 0}=11.67 \mathrm{meV}^{4}$ and was $\rho_{B i}=0.003378 \mathrm{GeV}^{4}$ at fiducial red shift $z=10^{12}$. The supernova observations fit an average

$$
\widetilde{w_{Q}}(N) \equiv \int_{0}^{N} w_{Q}\left(N^{\prime}\right) d N^{\prime} / N
$$

The CBR anisotropy and mass fluctuation spectrum, on the other hand, depend on "early quintessence" [9], back to the last scattering surface $z \sim 1100$. Where needed, we will fix $h^{2}=1 / 2$, so that the present critical density and smooth energy density are $\rho_{c r 0}=40.5 \mathrm{meV}^{4}, \rho_{Q 0}=28.8 \mathrm{meV}^{4}$ $=(2.3 \mathrm{meV})^{4}$.

This completes our discussion of the geometry of an accelerated flat universe. The next two sections review scalar field dynamics and the two attractor conditions, extending the many earlier optimistic treatments of the tracker condition [11-17] and of inverse power potentials (Sec. IV) $[18,19]$, by considering (1) poor trackers, (2) post-tracker behavior in the present quintessence-dominated era, (3) the range of initial conditions leading to tracking, and (4) the numerical problems encountered in cosmological dynamics, particularly in the freezing and tracking epochs. Sections IV and $\mathrm{V}$ conclude that the nearly static upper bound (8) requires that any quintessence must be either finely-tuned (crawling quintessence) or have large current curvature (cross-over quintessence [10]).

\section{DARK ENERGY AS A SCALAR FIELD}

\section{A. Quintessence dynamics: Potentials not yet truly slow-rolling}

Given that the GR universe is flat, presently dominated by smooth energy, and recently accelerated, is this smooth energy constant (a cosmological constant) or dynamic? While there is certainly no evidence for evolving smooth energy, attractive reasons for considering it to be dynamic are: (1) Theoretically, it can explain why the smooth energy density is now small but non-vanishing ("Why small?"), and can suggest reasons why the universe only recently became smooth energy dominated and expanding ("Why now?"); (2) Observationally, it may suggest the physical mechanism driving present cosmological evolution.

The smooth energy may be due to tangled topological defects, Chaplygin gases, trans-Planck or renormalization effects in ordinary curved space-time, or due to scalar fields with generic $[18,19]$ attractor properties, that make the present smooth energy density insensitive to a more-or-less broad range of unknown initial conditions.

The simplest dynamical realization for the smooth energy is by a spatially homogeneous light classical scalar field, minimally coupled to gravity, with zero true cosmological constant and kinetic energy. Canonical quintessence assumes an effective Lagrangian linear in the scalar field kinetic energy $K=\dot{\phi}^{2} / 2$, rolling down its self-potential $V(\phi)$ to zero. (At the end of this section, we briefly comment on k-essence, which assumes an effective Lagrangian that is non-linear in the kinetic energy.)

In canonical quintessence, the scalar field equation of motion 
TABLE I. Potentials described by roll $\lambda=-d \ln V / \varkappa d \phi$ and curvature $\eta=d^{2} V / V d(\varkappa \phi)^{2}$.

\begin{tabular}{|c|c|c|c|c|}
\hline$V(\phi)$ & $\lambda(\phi)$ & $\eta(\phi)=\lambda^{2} \Gamma$ & $\Gamma-1=1 / \beta(\phi)$ & NAME \\
\hline $\exp -\lambda \varkappa \phi$ & $\lambda=\sqrt{3 \gamma_{B} / \Omega_{Q}}$ & $\lambda^{2}=$ const $>3 \gamma_{B}$ & 0 & exponential \\
\hline $1 / \sinh ^{\alpha}(\tilde{\alpha} \varkappa \phi)$ & $(\alpha \tilde{\alpha}) \operatorname{coth}(\tilde{\alpha} \varkappa \phi)$ & $(\alpha \tilde{\alpha})^{2}\left[(1+\alpha) \operatorname{coth}^{2}(\tilde{\alpha} \varkappa \phi)-1\right]$ & $1 / \alpha \cosh ^{2}(\tilde{\alpha} \varkappa \phi)$ & $w_{Q}=-2 /(2+\alpha)$ \\
\hline$\phi^{-\alpha}$ & $\alpha / \varkappa \phi$ & $\alpha(\alpha+1) /(\varkappa \phi)^{2}$ & $1 / \alpha$ & inverse power \\
\hline const & 0 & 0 & $\infty$ & cosmological const \\
\hline$\phi^{-\alpha} \cdot \exp \frac{1}{2}(\varkappa \phi)^{2}$ & $\alpha / \varkappa \phi-\varkappa \phi$ & {$\left[\alpha(\alpha+1)+(1-2 \alpha)(\varkappa \phi)^{2}+(\varkappa \phi)^{4}\right] /(\varkappa \phi)^{2}$} & $\left(\alpha+(\varkappa \phi)^{2}\right) /\left(\alpha-(\varkappa \phi)^{2}\right)^{2}$ & SUGRA \\
\hline
\end{tabular}

$$
\ddot{\phi}+3 H \dot{\phi}+d V / d \phi=0
$$

has the first integral

$$
\rho_{Q}=\dot{\phi}^{2} / 2+V(\phi),
$$

where

$$
\begin{gathered}
V(N)=\rho_{Q}+d \rho_{Q} / 6 d N=\rho_{Q}\left(1-w_{Q}\right) / 2, \\
P / c^{2}=\dot{\phi}^{2} / 2-V(\phi) .
\end{gathered}
$$

The barotropic index

$$
\gamma_{Q}(N) \equiv-d \ln \rho_{Q} / 3 d N=\left(\left(\rho+P / c^{2}\right) / \rho\right)_{Q} \equiv 1+w_{Q}
$$

lies between 0 and 2, so that the weak energy condition, $\rho$ $+P / c^{2}>0, w>-1$, requires that the density $\rho$ and the Hubble expansion decrease monotonically. Because the scalar field does not cluster on supercluster scales, its mass must be $\lesssim 10^{-31} \mathrm{eV}$. Such an incredibly small mass is hard to protect against matter-coupling and SUSY-breaking interactions, unless the general relativity theory is expanded to a scalar-tensor theory of gravitation.

From the energy integral (11), the quintessence kinetic energy fraction of the total energy density $x^{2} \equiv \dot{\phi}^{2} / 2 \rho$ $=\gamma_{Q} \Omega_{Q} / 2=(\varkappa d \phi / d N)^{2} / 6$. The ratio of kinetic/potential energy $K / V=\left(1+w_{Q}\right) /\left(1-w_{Q}\right)$ has the rate of change $d \ln (K / V) / d N=6(\Delta-1), \quad$ where $\quad \Delta(N) \equiv-d \ln V / 3 \gamma_{Q} d N$ Thus, the roll,

$$
\lambda \equiv-d \ln V / x d \phi=\sqrt{3 \gamma_{Q} / \Omega_{Q}} \cdot \Delta,
$$

and

$$
d w_{Q} / d N=3\left(1-w_{Q}^{2}\right)(\Delta-1), \quad \varkappa d \phi / d N=\sqrt{3 \gamma_{Q} \Omega_{Q}}
$$

is a two-element non-autonomous system for the dependent variables $\phi, w_{Q}$. Integrating the second equation (15) implicitly relates $\phi$ and $V(\phi)$, so that, if the equation of state $w_{Q}(z)$ can be observed, the potential can be reconstructed. The overall equation of state is

$$
w=\gamma-1=w_{B} \Omega_{B}+w_{Q} \Omega_{Q},
$$

where the dimensionless ratios, $\Omega_{Q}, \Omega_{B} \equiv \rho_{B} /\left(\rho+\rho_{Q}\right)$, are the energy density fractions in quintessence and in the radiation-matter background, and $\gamma_{Q}, \gamma_{B} \equiv-d \ln \rho_{B} / 3 d N$ are their corresponding barotropic indices.

Defining the potential energy fraction $y^{2} \equiv V_{Q} / \rho$, Eqs. (15) have different scaling solutions whenever $w_{Q}$ or $K / V$ $\equiv(x / y)^{2}=\gamma_{Q} /\left(2-\gamma_{Q}\right) \approx$ const: kination, when $w_{Q} \approx 1, x$ $\gg y$; freezing, when $w_{Q} \approx-1, x \ll y$; tracking, when $\Delta$ $\approx 1, y \propto x$. The scale grows as $a \sim t^{2 / 3 \gamma_{B}}, t^{2 / 3} \gamma_{Q}$, in the radiation-matter background and in the quintessence eras respectively.

Besides the roll $\lambda$, the potential is characterized by the curvature

$$
\eta \equiv V^{\prime \prime} / V=\lambda^{2}-\lambda^{\prime}=\lambda^{2} \Gamma
$$

where

$$
\begin{gathered}
\Gamma \equiv V V^{\prime \prime} / V^{\prime 2} \equiv \eta / \lambda^{2} \equiv(1+1 / \beta), \quad 1 / \beta \equiv \Gamma-1=(1 / \lambda)^{\prime} \\
{ }^{\prime} \equiv d / \varkappa d \phi .
\end{gathered}
$$

When the roll is slow ( $\epsilon \equiv \lambda^{2} / 2 \ll 1$ ), the kinetic energy $\dot{\phi}^{2} / 2$ is negligible in the quintessence energy (12). When $\eta \ll 1, \ddot{\phi}$ is negligible in the equation of motion (10). In ordinary inflation, both these conditions hold (slow roll approximation): the expansion is dominated by the cosmological drag, and the field is nearly frozen.

In quintessence, on the other hand, the acceleration began only recently, when $\varkappa \phi_{0} \sim 1$, so that the roll $\lambda_{0}$ and curvature $\eta_{0}$ are still $\mathcal{O}(1)$, and their difference $\eta-\lambda^{2}=(\ln V)^{\prime \prime}$ $=-\lambda^{\prime}$ may be large or small, and their ratio $1<\Gamma=\eta / \lambda^{2}$ may be large. This invalidates the slow roll approximation for quintessence, so that the dynamical equations need to be integrated numerically. We ultimately handled the kinationfreezing and freezing-tracking transitions and numerical stability and round-off problems in the protracted frozen era, by implicit Adams backward differentiation procedures (Maple lsode[adamsfunc] and lsode[backfull]), with small adaptive step-size.

Unless it undergoes a symmetry-breaking phase transition, the quintessence potential rolls monotonically towards a minimum at $\phi=\infty$ or at some finite $\phi_{\min }$. A potential minimum realizes a large potential curvature. Either way, the potentials we consider are always convex. (Presumably, there is no true cosmological constant so that the potential energy and the Hubble expansion vanish asymptotically, avoiding the possibly worrisome future de Sitter event horizon, with attendant future causal isolation.) 
TABLE II. Tracker and present $\left(\Omega_{Q 0}=0.71\right)$ attractor solutions for inverse power and SUGRA potentials.

\begin{tabular}{|c|c|c|c|c|c|c|c|c|c|c|}
\hline$\alpha$ & $w_{Q t r}$ & $M$ & $\varkappa \phi_{0}$ & $\lambda_{0}$ & $\eta_{0}$ & $x_{0}^{2}$ & $y_{0}^{2}$ & $w_{Q 0}$ & $w_{Q 1}$ & $\Omega_{\text {Qimax }}$ \\
\hline 0 & -1 & $2.5 \mathrm{meV}$ & - & 0 (static) & 0 & 0 & .71 & -1 & 0 & $8 \times 10^{-45}$ \\
\hline 0.1 & $-0.937 \ldots-0.952$ & $12.1 \mathrm{meV}$ & 0.285 & 0.351 & 1.36 & .011 & .698 & -0.97 & 0.04 & $3 \times 10^{-36}$ \\
\hline 0.5 & $-0.733 \ldots-0.80$ & $4.8 \mathrm{eV}$ & 0.725 & 0.689 & 1.42 & .049 & .661 & -0.86 & 0.08 & $8 \times 10^{-10}$ \\
\hline 1 & $-0.555 \ldots-0.667$ & $2.2 \mathrm{keV}$ & 1.123 & 0.890 & 1.58 & .080 & .630 & -0.78 & 0.30 & $3 \times 10^{-2}$ \\
\hline 6 & $0 \ldots-0.25$ & 5.3 PeV & 4.029 & 1.489 (fast) & 2.59 & .201 & .509 & -0.41 & 0.40 & $3 \times 10^{-1}$ \\
\hline 1 & $-0.555 \ldots-0.667$ & $2.1 \mathrm{keV}$ & 0.870 & 0.280 & 2.40 & .024 & .685 & -0.82 & 0.72 & $3 \times 10^{-2}$ \\
\hline 6 & $0 \ldots-0.25$ & 3.0 PeV & 2.254 & 0.408 & 2.35 & .048 & .663 & -0.87 & 0.58 & $3 \times 10^{-1}$ \\
\hline
\end{tabular}

Both the roll and the curvature, $\lambda$ and $\eta$ are listed in Table I, for five different potentials. The third row gives the constant $\eta / \lambda^{2}$ or $\beta=$ const $\equiv \alpha$ potential. The first and fourth rows give its $\beta=0, \infty$ limits, the cosmological constant and exponential potentials. On the second row, where $\tilde{\alpha}$ $\equiv 1 / \sqrt{\alpha(2+\alpha)}$, the unrealistic constant $w_{Q}$ model interpolates between the inverse power potential, when $\tilde{\alpha} \varkappa \phi \ll 1$, and the exponential when $\tilde{\alpha} \varkappa \phi \gg 1$. The bottom row is the more realistic inflaton broken-SUSY supergravity (SUGRA) potential, in which, when $\phi \gtrsim M_{P}, \beta(\phi)$ decreases and the curvature $\eta(\phi)$ increases significantly

\section{B. Phaseportrait in terms of quintessence kinetic, potential energy canonical variables}

In place of the phase variables $\phi, w_{Q} \equiv(P / \rho)_{Q}$, we may use $x, y$, for which the equations of motion are [14-17]

$$
\begin{aligned}
d x / d N & =-3 x+\lambda \sqrt{3 / 2} y^{2}+3 x \gamma / 2 \\
d y / d N & =\lambda \sqrt{3 / 2} x y+3 y \gamma / 2 \\
d \lambda / d N & =-\sqrt{6} \lambda^{2} x / \beta \text { or } d(1 / \lambda) / d N \\
& =\sqrt{6} x / \beta .
\end{aligned}
$$

The overall equation of state of our two-component mixture of radiation-matter background and quintessence, $\gamma$ $=\gamma_{Q} \Omega_{Q}+\gamma_{B} \Omega_{B}=2 x^{2}+\gamma_{B}\left(1-x^{2}-y^{2}\right)$, is a timedependent function of the scalar field $\phi(N)$. Thus,

$$
\begin{gathered}
x^{2}+y^{2}=\Omega_{Q}, \quad 2 x^{2}=\Omega_{Q} \gamma_{Q}, \quad y^{2} / x^{2}=\left(1-w_{Q}\right) /\left(1+w_{Q}\right), \\
d \ln \left(x^{2} / y^{2}\right) / d N=6(\Delta-1) .
\end{gathered}
$$

The three-element system (19)-(21) is autonomous, except for the slow change in $\gamma_{B}(N)$ from $4 / 3$ to 1 , while gradually going from the radiation-dominated to the matter-dominated universe, around red-shift $z_{e q}=3454$.

The magnitude of $V$ needs to be fine-tuned to the present value $V_{0}=\rho_{c r 0} y_{0}^{2}=\rho_{Q 0}\left(1-w_{Q 0}\right) / 2$. For example, inverse power potentials, require the energy scales $M_{\alpha}$ $=\left(V_{0} \phi_{0}^{\alpha}\right)^{1 /(4+\alpha)}$, listed in the third column of Table II. For shallow potentials $(\alpha<0.2)$, this energy scale is close to observed neutrino masses and to the present radiation temperature, possibly suggesting some role for the neutrino mass mechanism or for the matter-radiation transition, in bringing about quintessence dominance. For steep potentials ( $\alpha$ $>1$ ), this mass scale can be considerably larger, suggesting the larger scales we encounter in particle physics.

While the evolution of a homogeneous scalar field depends only on its equation of state $w_{Q}=\left(P / \rho c^{2}\right)_{Q}$, the growth of its fluctuations depends also on the quintessence sound speed $c_{s}^{2}=(d P / d \dot{\phi}) /\left(d \rho_{Q} / d \dot{\phi}\right)$. With the linear form for the kinetic energy $K=\dot{\phi}^{2} / 2$ that canonical quintessence assumes, $c_{s}^{2}=c^{2}$ and $-1 \leqslant w_{Q} \leqslant 1, d w_{Q} / d z>0$. Noncanonical scalar fields, such as k-essence [20,21], permit $w_{Q}<-1, d w_{Q}(z) / d z<0$ and give different sound speed and structure evolution.

Effective actions with such non-linear kinetic energy appear in some string and supergravity theories. Despite this possible difference in sign of $d w_{Q} / d z$, unless $c_{s}^{2} \sim 0$ since the surface of last scattering, $\mathrm{k}$-essence is hardly distinguishable from quintessence [22]. Although tracking trajectories in the radiation-dominated era, are quickly attracted towards $w_{Q} \approx-1$ in the matter-dominated era, k-essence, like quintessence, requires fine-tuning. The most catastrophic consequence of violating the weak energy condition is that the scalar field energy can grow with time, ultimately (after about 35 Gyr of scalar field dominance [23]) ripping apart bound systems, such as galaxies, planets and atoms.

\section{ATTRACTORS IN TRACKING AND IN QUINTESSENCE ERAS}

We will consider only canonical quintessence evolution with the Friedmann expansion rate (2). The scalar field equations of motion enjoy a fixed attractor solution $\lambda=$ const (exponential potential), when $\beta=\infty$, and two different stable instantaneous attractor solutions $[12,13,16,19]$, when $\lambda$ is slowly varying in Eq. (21), because $-\lambda^{\prime}=\eta-\lambda^{2} \ll 1, \Gamma$ $\gtrsim 1$. Depending on the roll or $t \equiv \lambda^{2} / 3$, these are: The slowroll ("future") attractor

$$
x_{c}^{2}(\lambda)=t / 2, \quad y_{c}^{2}(\lambda)=1-t / 2, \quad \Omega_{Q c}=1, \quad t<2 .
$$

The fast-roll ("past") attractor

$$
\begin{gathered}
x_{c}^{2}(\lambda)=\gamma_{Q}^{2} / 2 t, \quad y_{c}^{2}(\lambda)=\gamma_{Q}\left(2-\gamma_{Q}\right) / 2 t, \\
\Omega_{Q c}=\gamma_{Q} / t, \quad t>\gamma_{Q},
\end{gathered}
$$

where the equation of state [17] is 


$$
\begin{aligned}
\gamma_{\text {Qatt }}(\lambda)= & \frac{1}{2}\left[\left((1+2 / \beta) t+\gamma_{B}\right)\right. \\
& \left.-\sqrt{\left((1+2 / \beta) t-\gamma_{B}\right)^{2}+8 \gamma_{B} t / \beta}\right] \\
= & \begin{cases}0, & \beta=0 \\
t, & t \ll 1 \\
\gamma_{B} \beta /(\beta+2), & t \gg 1 \\
\gamma_{B}, & \beta=\infty .\end{cases}
\end{aligned}
$$

Tracking quintessence $[12,18,19]$ exploits the fast-roll attractor to explain the very small present smooth energy density dynamically ("Why small?"). Instead of a finely tuned cosmological constant, quintessence requires a potential strength fine-tuned to the present time ("Why now?"). We reserve the term tracker for these fast-roll attractors in the radiation or matter background-dominated era, for which $\beta$ and $\gamma_{Q a t t}=2 \gamma_{B} \beta /(2+\beta)$ are nearly constant. The tracker condition can then be written as

$$
\Delta=\lambda / \lambda_{t r}=\sqrt{\gamma_{Q} / \gamma_{Q t r}} \approx 1, \quad|d \ln \beta / d N| \ll 1,
$$

where $\lambda_{t r} \equiv \sqrt{3 \gamma_{Q a t t} / \Omega_{Q}}$. The quintessence fraction scales as $\Omega_{\text {Qatt }} \sim a^{\left.6 \gamma_{B} / \tau \beta+2\right)} \sim t^{P}$, where $P \equiv 4 /(2+\beta)$. In good trackers $\left(\beta \gg 1, \gamma_{Q a t t} \lesssim \gamma_{B}\right), \Omega_{Q}$ and $\lambda_{t r}$ vary slowly. Indeed, very steep $(\beta \gg 1)$ potentials approximate exponential potentials and track for a very long time, with slowly decreasing $\lambda$ and increasing $\Omega_{Q}$. They show early quintessence because, from $\lesssim 0.05$ at nucleosynthesis, $\Omega_{Q}(z)$ slowly increases and is already non-negligible at recombination and structure formation. In poor trackers $\left(\beta<1, \gamma_{Q t r}\right.$ $<\gamma_{B} / 3$ ), the roll is slow, $\rho_{Q t r}$ is slowly decreasing ("crawling"), and $\Omega_{Q} \sim t^{2}, \lambda_{t r} \sim t^{-1}$ are changing with time.

At present, the roll is no longer fast and $\gamma_{Q}(\lambda)$ is decreasing, curving the attractor trajectories towards the y-axis. We therefore need to study the quintessence-dominant posttracking era when $\Delta-1=d \ln (K / V) / 6 d N<0, w_{Q} \rightarrow-1$. Figure 1 shows the instantaneous attractor phaseportraits, while tracking and while quintessence-dominated, for two inverse power (solid) and two SUGRA (dotted) potentials, all fitted to reach $\Omega_{Q 0}=0.71$, at the present time. The constant $w_{Q}$ $=-0.67,-0.25$ trajectories are shown dashed. For each form of potential, the shallow potential $\alpha=1$ attractor trajectories appear on the left, the steep potential $\alpha=6$ attractor trajectories on the right. The cosmological constant trajectory $\alpha=0$ is the y-axis. The exponential potential has fixed $\Omega_{Q}$; its fixed point, $x=\sqrt{\Omega_{Q 0} / 2}=y$, would fall on the circle $\Omega_{Q 0}=0.71$, just off the right of the figure.

Along these trajectories, evolution is measured by the quintessence fraction $\Omega_{Q}=x^{2}+y^{2}$, which is zero in the distant past (lower left corner), unity in the far future (top of phaseportrait), and has been fitted to $\Omega_{Q 0}=0.71$ at the present time. In the background-dominated era, all phase trajectories track with nearly constant slope $(y / x)_{t r}=\sqrt{V / K}$ gradually increasing from $\sqrt{1 / 2+3 / \beta}$ in the radiationdominated era to $\sqrt{1+4 / \beta}$ in the matter-dominated era, and equation of state $w_{Q t r}=-2 /(\beta+2)$. Later, as quintessence grows, the three potential forms curve differently towards the

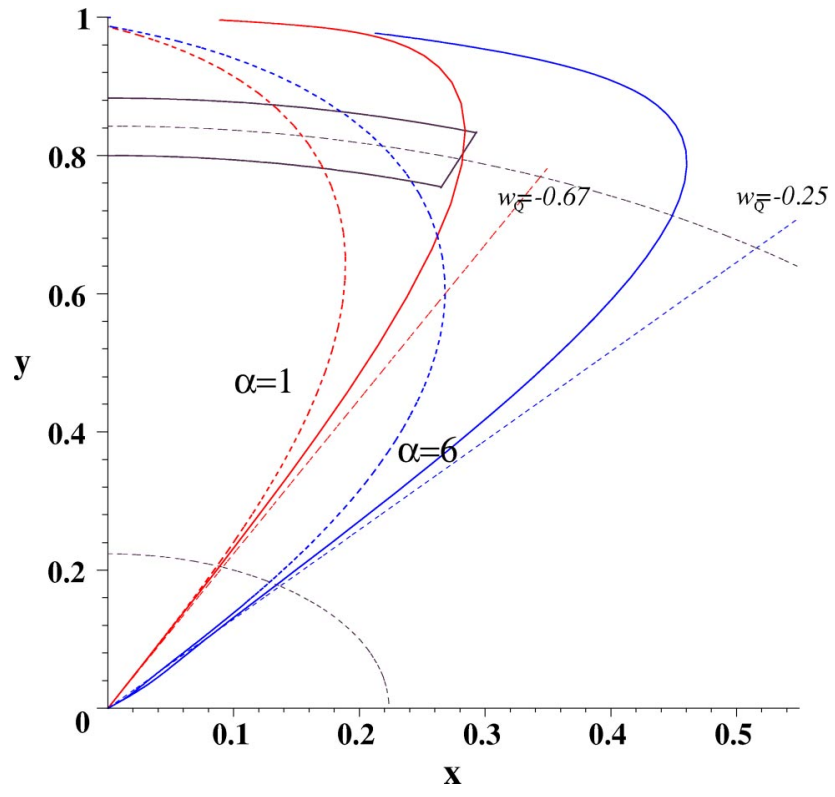

FIG. 1. (Color online) Complete phase portraits of instantaneous attractors, from red shift $z=10^{12}$ in lower left corner, to $z=10^{-10}$ on the top, now passing through $\Omega_{Q 0}=0.71$. The evolution is shown, on the right for three steep potentials $(\alpha=6)$; on the left, for three shallow potentials $(\alpha=1)$. The evolution of each model is shown against the dashed circles $\Omega_{Q}=x^{2}+y^{2}=0.71$ at present, $=0.1$ when quintessence became significant. In the backgrounddominated era $\Omega_{Q} \ll 1$ (lower left), all attractors track with $y \propto x$ and $w_{Q t r}=-0.25,-0.67$ for $\alpha=6,1$ respectively. When quintessence begins dominating $\Omega_{Q}>0.1$, the inverse power trajectories (solid), start late to curve slowly towards the y-axis $\left(w_{Q}=-1\right)$; both SUGRA trajectories (dotted), start early to curve rapidly towards the y-axis. The constant $w_{Q}$ trajectories are shown dashed. The trapezoidal region on the upper left is the observationally allowed present phase space.

y-axis: the constant $w_{Q}$ potentials have been chosen to make their trajectories hold their tracker values; the SUGRA potential phase trajectories curve strongly towards the $y$-axis. The presently-observed trapezoidal region in phase space nearly excludes both inverse power potential, but comfortably allows the SUGRA potential for a range in parameter $\alpha$.

The bottom half of Table II summarizes, on rows five and six, seven and eight, the present properties of the $\alpha=6,1$ inverse-power and SUGRA phase trajectories respectively. (The top half of Table I also includes, on row 2, the cosmological constant phase trajectory, which is the y-axis in Fig. 1 . Rows $3-4$, summarize the nearly static $\alpha=0.5,0.1$ phase trajectories, which are not plotted in Fig. 1.) For each $\alpha$, the second column tabulates the range in tracker equation of state $w_{Q t r}$, running from the early radiation-dominated era, to the late matter-dominated era. To the right of the double line, columns 3-6, give the potential energy scale $M_{\alpha}$ needed to reach the present $\Omega_{Q 0}=0.71$, and the present values of the quintessence field, roll, and curvature. Columns seven and eight give the quintessence kinetic and potential energy fractions composing the total quintessence energy fraction $\Omega_{Q 0}$. Column nine shows that only the highcurvature SUGRA potentials give $w_{Q 0}<0.78$, as observationally required. If, in place of a cosmological constant, 


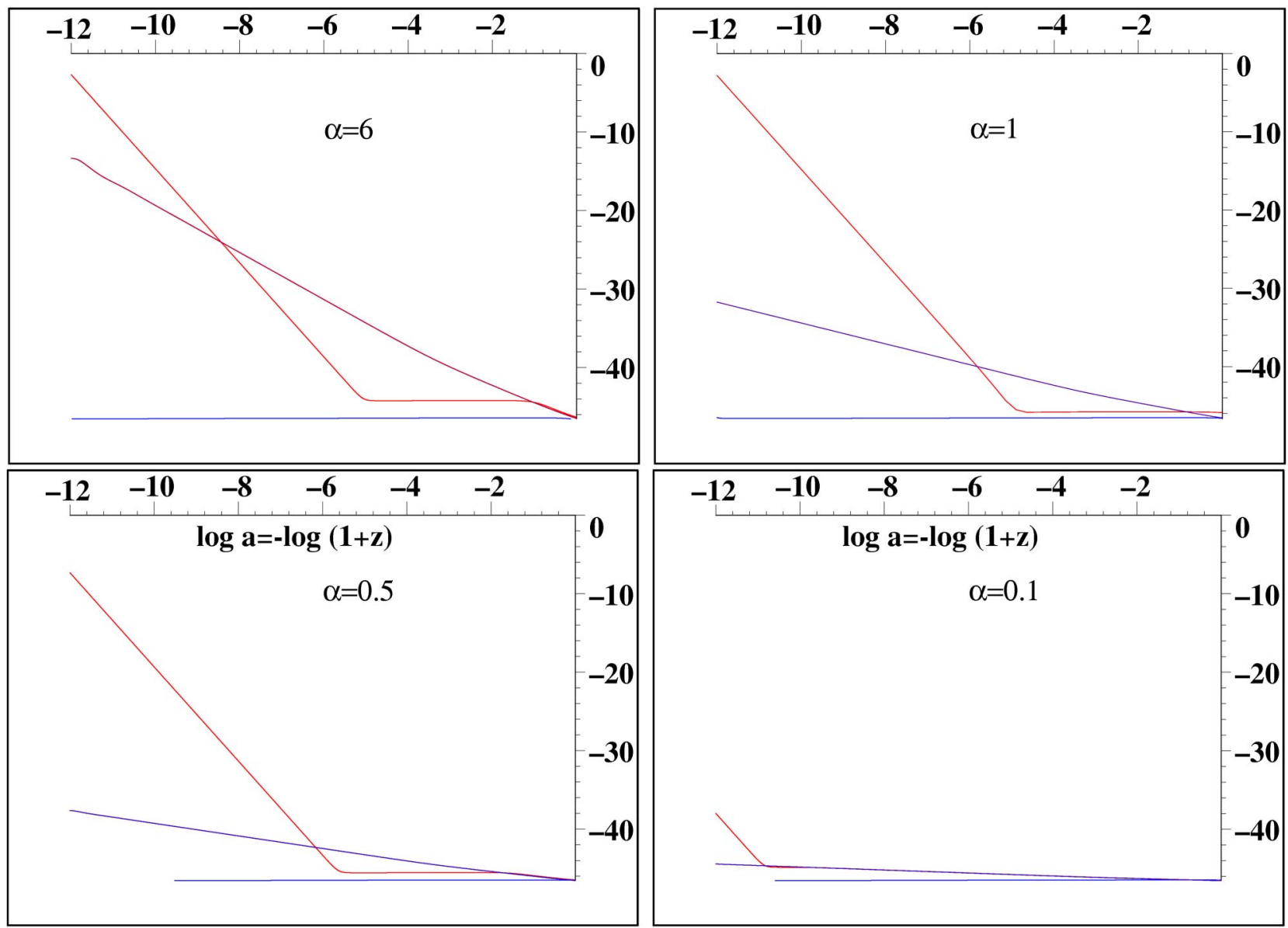

FIG. 2. (Color online) Evolution of quintessence energy density, $\log \rho_{Q}\left(\mathrm{GeV}^{4}\right)$, on vertical axes, for four inverse power potentials $\alpha$ $=6,1,0.5,0.1$, from red-shift $z=10^{12}$, to the present value $\rho_{Q 0}=0.71 \rho_{c r 0}$. In all figures, the central trajectory is the attractor, starting with tracker slope $d \ln \rho_{Q} / d N=-6 \gamma_{B} /(2+\alpha)$. The lower curve is the maximal undershoot trajectory, which freezes immediately and then crawls slowly to join the attractor now. The upper curve is the maximal overshoot trajectory, which kinates with slope -6 , before freezing late and now reaching the attractor. Poor trackers freeze early, out of a narrow range in $\log \rho_{Q i}$.

quintessence potentials like these exist, then the present redshift variation $w_{Q 1} \equiv\left((1+z) d w_{Q}(z) / d z\right)_{0}$ in column ten may ultimately be observable. The last column gives the upper bound to the basin of attraction, to be discussed in the next section.

\section{GOOD TRACKERS MUST NOW ROLL-OVER TO SLOW ROLL}

\section{A. Basin of attraction for trajectories flowing onto the tracker}

To obtain the presently small smooth energy without finetuning initial conditions, phase trajectories must flow onto the attractor before now and for a broad range of initial conditions, the basin of attraction. A good tracker starts from a broad basin of attraction and can still freeze relatively late, before tracking with appreciable kinetic energy $K \lesssim V$. A poor tracker starts from a narrow basin of attraction and freezes early and for a long time, before tracking with small kinetic energy $K \ll V$.

Tracking is always preceeded by freezing, which begins when the curvature drops, while the roll is still appreciable. From any undershoot initial conditions $\rho_{Q 0}<\rho_{Q i}<\rho_{Q t r}$, freezing starts directly. (Indeed, if the initial density $\rho_{Q i}$ is small enough, the field crawls down to its present value $\rho_{Q 0}$, without ever tracking.) But, starting from overshoot initial conditions $\Omega_{O i}>\Omega_{Q t r}$, freezing starts much later, at a value $\phi_{f r} \gtrsim M_{P} \sqrt{6 \Omega_{Q i}}$, only after a long kinated era $(x \gg y)$, during which $\dot{\phi}^{2} \approx 2 \rho_{Q}$ lets the field grow rapidly while $\Omega_{Q}$ decreases. Above some $\Omega_{\text {Qimax }}$, too much overshoot would make the phase trajectories freeze so late as to reach the attractor only in the future. We will now extend earlier treatments $[12,13]$ of trackers to solutions that reach the attractor only now, in the present, quintessence-dominated era.

\section{B. Inverse power potentials do not show enough present curvature}

For the simple inverse power law potentials

$$
V(\phi)=M_{\alpha}^{4+\alpha} / \phi^{\alpha},
$$

$\beta(\phi)=$ const $\equiv \alpha$, so that the equation of motion (10) has exactly scaling solutions in both the radiation- and the matter-dominated eras. These much-studied potentials are in- 
TABLE III. Quintessence potentials which track early, but slow-roll now.

\begin{tabular}{lcc}
\hline \hline Potential $V(\phi)$ & Theoretical origin & References \\
\hline$M^{4}[\cos (\phi / f)+1]$ & String, M-theory pseudo Nambu-Goldstone light & {$[11,33-37]$} \\
$M^{4+\alpha} \phi^{-\alpha} \cdot \exp \frac{1}{2}(\varkappa \phi)^{\beta} / 2$ & axion & \\
$M_{P}^{4}\left[A+\left(\varkappa \phi-\varkappa \phi_{m}\right)^{\alpha}\right] \exp (-\lambda \varkappa \phi)$ & SUGRA, minimum at $(\varkappa \phi)^{\beta}=2 \alpha / \beta$ & {$[24-26]$} \\
& Exponential modified by prefactor, to give local & {$[38,39]$} \\
\hline \hline
\end{tabular}

teresting, because they approximate any potential, while tracking. They arise naturally in supersymmetric condensate models for QCD or instanton SUSY-breaking [24-26], but acquire appreciable quantum corrections when $\phi \geq M_{P}$. For these potentials, $\lambda \sim V^{1 / \alpha} \sim(y H)^{2 / \alpha}$, the third equation (19) integrates to $\lambda=\lambda_{0}\left(y H / y_{0} H_{0}\right)^{2 / \alpha}$ in terms of present values of $y, H, \lambda$.

The second column in Table II gives the range in tracker values $w_{Q t r}$, from $(\alpha-6) / 3(\alpha+2)$, during the radiationdominated era, to $-2 /(\alpha+2)$, during the matter-dominated era. The third column tabulates the quintessence energy scales $M_{\alpha}$ needed, in order to fit $\Omega_{Q 0}=0.71$.

After tracking, these trajectories curve towards the $x=0$ (asymptotic de Sitter) axis. Between the two vertical double bars, columns four through nine, summarize the present (post-tracker) values for trajectories that have tracked before now. The steep $\alpha=6$ trajectory would by now track down only to $w_{Q 0}=-0.41$, which is excluded observationally by the constraint (8), which requires $\alpha \lesssim 1$ [12]. Inverse power trackers that are now approximately slow-rolling $\left(\lambda_{0}<1\right)$ could only have been reached from a narrow tracking basin of attraction. Indeed, in the static limit (cosmological constant), the present smooth energy must arise out of the unique initial condition $\rho_{Q i}=\rho_{Q 0}$.

Integrating Eqs. (17), (18), the last column tabulates, for each $\alpha$, the maximum initial quintessence fraction that would track and finally reach the presently-allowed value $\Omega_{Q 0}=0.71$. Likewise, the top curves in each of Fig. 2 show, on a logarithmic scale, the maximum initial quintessence energy density that would finally reach $\rho_{B 0}=28.8 \mathrm{meV}^{4}$. For large $\alpha$, the quintessence potential would now still be fastrolling [8]. As $\alpha$ decreases, $w_{Q 0}$ decreases, but the basin of attraction shrinks. For $\alpha<0.5$, the range in initial values $\Omega_{Q i}$ that track before now is already almost 10 orders of magnitude narrower than the initial range of the good $\alpha=6$ trackers first considered [12]. For a cosmological constant ( $\alpha$ $=0$ ), the present vacuum energy is realized only if it is initially tuned uniquely to its present value $\rho_{Q 0}$ $=28.8 \mathrm{meV}^{4}$.

Because the observed $\widetilde{w_{Q}}$ is already close to the cosmological constant value -1 , an inverse power potential requires $\alpha<1$, so that the potential energy always dominated the kinetic energy $(x \ll y)$. These nearly flat trajectories never track, but "crawl" [27] towards their present values, only because they were initially tuned close to these values.

\section{SUGRA potentials now curve over towards slow roll}

A good tracker with large basin of attraction must, while tracking, have small potential curvature. But, if it is to slow roll down to $\widetilde{w_{Q}}<-0.78$, after tracking, it must develop a large potential curvature $\eta[\beta(\phi)$ must decrease, $P(\phi)$ increase], so that $\Omega_{Q} \sim t^{P}$ grows rapidly at late times [12]. Such cross-over quintessence [9] is characterized by $w_{Q}(z)$ reducing in the recent past $(z<0.5)$.

The roll-over behavior needed is illustrated in the popular potentials listed in Table III. (A longer list of potentials is given in Refs. [28-32].) For example, the SUGRA models on the bottom row of Table I have minima at $\varkappa \phi=\sqrt{\alpha}$, beyond which the curvature $\eta$ increases, allowing $\beta$ to decrease precipitously from $\alpha$ in the tracking era, to $\mathcal{O} \sim(\alpha$ $-1)^{2} /(\alpha+1)$ at present. After tracking in the backgrounddominated era, these SUGRA phase trajectories, summarized on the bottom two rows of Table II and dotted in Fig. 1, curve over towards lower $w_{Q 0}$ values, in marginal agreement with observations, for a large range in $\alpha$ values.

\section{CONCLUSION: CANONICAL QUINTESSENCE REQUIRES TWO COSMIC COINCIDENCES}

We have not considered modified gravity, quantum corrections to classical general relativity, topological defects, non-canonical scalar fields, a true cosmological constant, nor matter-coupled quintessence. But, within canonical quintessence, the observations allow phase trajectories that are insensitive to initial conditions, only if the potential's curvature increases rapidly, just before the present epoch.

Difficult combined supernova, CBR and cosmic shear observations in the next decades may yet tell whether the smooth energy is static or dynamic and, if dynamic, whether it is quintessence, k-essence or even not driven by a scalar field. Otherwise, a dynamic smooth energy appears hardly distinguishable, theoretically and phenomenologically, from the small cosmological constant it was designed to explain.

The original cosmological coincidence problem was to understand why the smooth energy density is now so small, after allowing large scale structure formation, necessary prerequisites of life and consciousness. This coincidence problem is now compounded by the requirements that the quintessence potential energy now be small and its curvature now be fast-increasing. In two ways, recent cosmological observations distinguish the special time in which we live, in support of weak anthropic reasoning in cosmology.

\section{ACKNOWLEDGMENT}

We are indebted to US Department of Energy grant DEFGO2-95ER40893 at the University of Pennsylvania for partial travel support. 
[1] I. Moar, R. Brustein, J. McMahon, and P.J. Steinhardt, Phys. Rev. D 65, 123003 (2002).

[2] T. Nakamura and T. Chiba, Mon. Not. R. Astron. Soc. 306, 696 (1999).

[3] D. Huterer and M.S. Turner, Phys. Rev. D 60, 081301 (1999); 64, 123527 (2001).

[4] J.A. Frieman, D. Huterer, E.V. Linder, and M.S. Turner, Phys. Rev. D 67, 083505 (2003).

[5] T.R. Choudhury and T. Padmanabhan, Mon. Not. R. Astron. Soc. (to be published).

[6] D.N. Spergel et al., Astrophys. J., Suppl. Ser. 148, 175 (2003).

[7] J.L. Tonry et al., Astrophys. J. 594, 1 (2003).

[8] S. Bludman and M. Roos, Astrophys. J. 547, 77 (2001).

[9] R.R. Caldwell, M. Doran, C.M. Mueller, G. Schaefer, and C. Wetterich, Astrophys. J. Lett. 591, L75 (2003).

[10] C. Wetterich, Astropart. Phys. 10, 2 (2003).

[11] R.R. Caldwell, R. Dave, and P.J. Steinhardt, Phys. Rev. Lett. 80, 1582 (1998).

[12] P.J. Steinhardt, L. Wang, and I. Zlatev, Phys. Rev. D 59, 123504 (1999).

[13] I. Zlatev, L. Wang, and P.J. Steinhardt, Phys. Rev. Lett. 82, 896 (1999).

[14] P. Ferreira and M. Joyce, Phys. Rev. Lett. 79, 4740 (1997); Phys. Rev. D 57, 6022 (1998).

[15] A.R. Liddle and R.J. Scherer, Phys. Rev. D 59, 023509 (1999).

[16] E.J. Copeland, A.R. Liddle, and D. Wands, Phys. Rev. D 57, 4686 (1998).

[17] S.C.C. Ng, N.J. Nunes, and F. Rosati, Phys. Rev. D 64, 083510 (2001).

[18] B. Ratra and P.J.E. Peebles, Phys. Rev. D 37, 3406 (1988).
[19] C. Wetterich, Nucl. Phys. B302, 668 (1988).

[20] C. Armendariz-Picon, V. Mukhanov, and P.J. Steinhardt, Phys. Rev. Lett. 85, 4438 (2000); Phys. Rev. D 63, 103510 (2001).

[21] J.K. Erikson, R.R. Caldwell, P.J. Steinhardt, C. ArmendarizPicon, and V. Mukhanov, Phys. Rev. Lett. 88, 121301 (2002).

[22] V. Barger and D. Marfata, Phys. Lett. B 498, 67 (2001).

[23] R.R. Caldwell, M. Kamionkowski, and N.N. Weinberg, Phys. Rev. Lett. 91, 071301 (2003).

[24] P. Binutray, Phys. Rev. D 60, 063502 (1999).

[25] Ph. Brax and J. Martin, Phys. Lett. B 468, 40 (1999).

[26] Ph. Brax, J. Martin, and A. Riazuelo, Phys. Rev. D 64, 083505 (2001).

[27] G. Huey and J.E. Lidsey, Phys. Lett. B 514, 217 (2001).

[28] J. Weller and A. Albrecht, Phys. Rev. D 65, 103512 (2002).

[29] V. Sahni, Chaos 16, 527 (2003).

[30] P.J.E. Peebles and B. Ratra, Rev. Mod. Phys. 75, 559 (2003).

[31] V. Sahni and A. Starobinski, Int. J. Mod. Phys. D 9, 373 (2000).

[32] T. Padmanabhan, Phys. Rep. 380, 235 (2003).

[33] K. Choi, Phys. Rev. D 62, 043509 (2000).

[34] N.J. Nunes and E.J. Copeland, Phys. Rev. D 66, 043524 (2002).

[35] A. Albrecht, C.P. Burgess, F. Ravndal, and C. Skordis, Phys. Rev. D 65, 123507 (2002).

[36] J. Frieman, C.T. Hill, A. Stebbins, and T. Waga, Phys. Rev. Lett. 75, 2077 (1995).

[37] K. Coble, S. Dodelson, and J.A. Frieman, Phys. Rev. D 55, 1851 (1997).

[38] A. Albrecht and C. Skordis, Phys. Rev. Lett. 84, 2076 (2000).

[39] C. Skordis and A. Albrecht, Phys. Rev. D 66, 043523 (2002). 